\title{
Stability of up-milling and down-milling, part 1: alternative analytical methods
}

\author{
T. Insperger a, B.P. Mann ${ }^{\text {b }}$, G. Stépán ${ }^{\mathrm{c}, *}$, P.V. Bayly ${ }^{\mathrm{d}}$ \\ a Department of Applied Mechanics, Budapest University of Technology and Economics, Budapest, H-1521, Hungary \\ ${ }^{\mathrm{b}}$ Department of Mechanical Engineering, Washington University, 1 Brookings Drive, St. Louis, MO 63130, USA \\ c Department of Applied Mechanics, Budapest University of Technology and Economics, Budapest, H-1521, Hungary \\ ${ }^{\mathrm{d}}$ Department of Mechanical Engineering, Washington University, 1 Brookings Drive, St. Louis, MO 63130, USA
}

Received 25 June 2002; accepted 20 August 2002

\begin{abstract}
The dynamic stability of the milling process is investigated through a single degree of freedom mechanical model. Two alternative analytical methods are introduced, both based on finite dimensional discrete map representations of the governing time periodic delay-differential equation.

Stability charts and chatter frequencies are determined for partial immersion up- and down-milling, and for full immersion milling operations. A special duality property of stability regions for up- and down-milling is shown and explained.

(C) 2002 Elsevier Science Ltd. All rights reserved.
\end{abstract}

Keywords: High-speed milling; Regenerative effect; Tooth pass excitation

\section{Introduction}

Increased industrial competition has driven the need for manufacturers to reduce costs and increase dimensional accuracy. Machining operations are one of the most widely used manufacturing processes [1]. The efficiency of a machining operation is dictated by the metal removal rates, cycle time, machine down time and tool wear. Optimisation of these parameters without sacrificing part quality is of key importance. A primary factor that limits process optimisation in machining is a phenomenon called chatter. Chatter is a dynamic instability that can limit material removal rates, cause a poor surface finish and potentially damage the tool and the workpiece.

The history of machine tool chatter goes back almost 100 years, when Taylor [2] described machine tool chatter as the "most obscure and delicate of all problems facing the machinist". After the extensive work of Tlusty et al. [3], Tobias [4] and Kudinov [5,6], the so-called

\footnotetext{
* Corresponding author. Tel.: +36-1-463-1369; fax: +36-1-4633471.

E-mail address: stepan@mm.bme.hu (G. Stépán).
}

regenerative effect has become the most commonly accepted explanation for machine tool chatter [7-10]. This effect is related to the wavy workpiece surface generated by the previous cutting tooth passage. The corresponding mathematical models are delay-differential equations (DDEs) with infinite dimensional state spaces.

For continuous cutting operations, like turning, the governing equation is autonomous, and stability conditions can be given in closed form $[1,11,12]$. The study of nonlinear phenomena in the cutting process showed that the chatter frequencies are related to unstable periodic motions about the stable stationary cutting, i.e. a so-called subcritical Hopf bifurcation occurs, as it was proved experimentally by Shi and Tobias [13] and later analytically by Stépán and Kalmár-Nagy [14].

In the case of milling, the direction of the cutting force is changing due to the tool rotation, and the cutting is also interrupted as each tooth enters and leaves the workpiece. Consequently, the resulting equation of motion is a DDE with a time periodic coefficient. The Floquet theory of periodic ordinary differential equations (ODEs) can be extended for these systems [15,16], and the stability properties are determined by the eigenvalues of the monodromy operator of the systems. These eigen- 
values are the so-called characteristic multipliers. The monodromy operator can be represented by an infinite dimensional matrix. This causes difficulties, of course, when trying to obtain closed form stability predictions. Usually, a finite dimensional approximate transition matrix is used to predict stability properties. Several analytical methods have been developed to determine the stability boundaries for milling [11,17-23]. Numerical simulation can also be used to capture the interrupted nature of the milling process [24-28], but the exploration of parameter space via time domain simulation is inefficient.

Analytical investigations have predicted the occurrence of new bifurcation phenomena in interrupted cutting processes. In addition to Hopf bifurcations, period doubling bifurcations are also a typical form of instability, as it was shown analytically by Insperger and Stépán [21], Corpus and Endres [29], Bayly et al. [22], Davies et al. [20], via numerical simulation by Zhao and Balachandran [26], and confirmed experimentally by Bayly et al. [22] and Davies et al. [20]. The nonlinear analysis of Stépán and Szalai [30] showed that this period doubling bifurcation is also subcritical.

In this paper, two analytical methods are introduced for stability prediction of general milling operations: the finite element analysis in time (FEAT) method and the semi-discretization (SD) method. Both methods form a finite dimensional transition matrix as an approximation of the infinite dimensional monodromy operator. The FEAT method presented in this paper is an extension of the method developed by Bayly et al. [22] for an interrupted turning process. The current analysis is different because it models milling more closely by including the changing direction of cutting forces. The SD method, first introduced by Insperger and Stépán [31], is also applied to milling. Due to the complicated and not fully explored structure of the stability charts, the comparison of the results of the two basically different approximation methods gives validity to the calculations. The analyses are carried out for various radial immersions of up-milling and down-milling for a single degree of freedom (SDOF) mechanical model. Stability predictions show that the regions of instability for up-milling and down-milling are about reversed at low immersions. Experimental evidence is given to confirm stability predictions in Section 2 of the paper.

\section{Mechanical model}

A schematic diagram of the milling process is shown in Fig. 1. The structure is assumed to be flexible in the $x$-direction only, so the system can be treated as SDOF. A summation of cutting forces acting on the tool produces the following equation of motion:

$\ddot{x}(t)+2 \zeta \omega_{n} \dot{x}(t)+\omega_{\mathrm{n}}^{2} x(t)=\frac{F_{x}}{m}$,

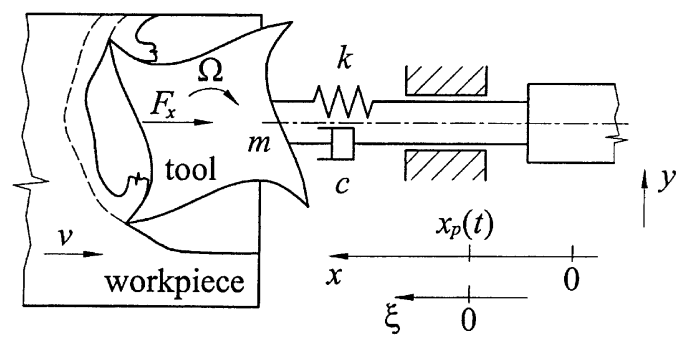

Fig. 1. SDOF mechanical model of the milling process.

where $m$ is the modal mass, $\zeta$ is the damping ratio, $\omega_{n}$ is the natural angular frequency, and $F_{x}$ is the cutting force in the $x$-direction for a zero helix cutter. According to Fig. 2, the $x$ component of the cutting force on the $p^{\text {th }}$ tooth is given by:

$F_{x p}=g_{p}(t)\left(-F_{t p} \cos \theta_{p}-F_{n p} \sin \theta_{p}\right)$,

where $g_{p}(t)$ acts as a switching function. It is equal to one if the $p^{\text {th }}$ tooth is active and zero if it is not cutting [11,17]. The tangential and normal cutting force components are considered to be the product of the tangential and normal linearised cutting coefficients $K_{t}$ and $K_{n}$, respectively, the nominal depth of cut $b$, and the instantaneous chip width $w_{p}$ :

$F_{t p}=K_{t} b w_{p}$,

$F_{n p}=K_{n} b w_{p}$,

where $w_{p}$ depends on the feed per tooth $h$, the cutter angle $\theta_{p}$, and the regeneration in the flexible direction of the structure, as follows:

$w_{p}(t)=h \sin \theta_{p}(t)+[x(t)-x(t-\tau)] \sin \theta_{p}(t)$.

Here $\tau=60 / N \Omega[\mathrm{s}]$ is the tooth pass period, $\Omega$ is the spindle speed given in rpm and $N$ is the number of teeth.

A summation over the total number of cutting teeth $N$, and the substitution of Eqs. (3-5) into eq. (2) yield the total cutting force acting in the $x$-direction:

$$
\begin{aligned}
F_{x}(t) & =\sum_{p=1}^{N} g_{p}(t)\left[-K_{t} b \cos \theta_{p}-K_{n} b \sin \theta_{p}\right]\left\{h \sin \theta_{p}(t)\right. \\
+ & {\left.[x(t)-x(t-\tau)] \sin \theta_{p}(t)\right\} . }
\end{aligned}
$$

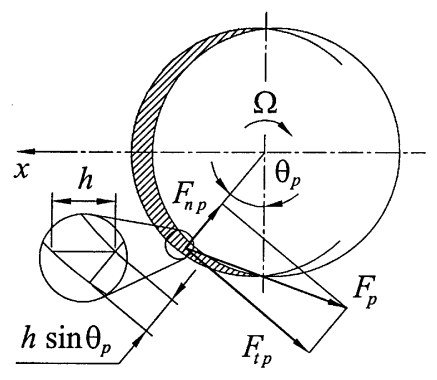

Fig. 2. Cutting force components in milling process. 
The angular position of the tool reads $\theta_{p}(t)=$ $(2 \pi \Omega / 60) t+p 2 \pi / N$, where $\Omega$ is given in rpm. A reduction in notation is achieved by substitution of the following terms:

$$
\begin{aligned}
& K_{s}(t)=\sum_{p=1}^{N} g_{p}(t)\left[K_{t} \cos \theta_{p}(t)+K_{n} \sin \theta_{p}(t)\right] \sin \theta_{p}(t), \\
& f_{0}(t)=\sum_{p=1}^{N} g_{p}(t)\left[K_{t} \cos \theta_{p}(t)+K_{n} \sin \theta_{p}(t)\right] h \sin \theta_{p}(t) .
\end{aligned}
$$

The functions $K_{s}(t)$ and $f_{o}(t)$ are $\tau$-periodic. The resulted equation of motion becomes:

$$
\begin{aligned}
\ddot{x}(t) & +2 \zeta \omega_{n} \dot{x}(t)+\omega_{n}^{2} x(t)=-\frac{b K_{s}(t)}{m}[x(t)-x(t-\tau)] \\
& -\frac{b f_{0}(t)}{m} .
\end{aligned}
$$

Here, the term $b f_{o}(t) / m$ can be eliminated from the equation. Assume the solution of the form:

$x(t)=x_{p}(t)+\xi(t)$

where $x_{p}(t)=x_{p}(t+\tau)$ is a $\tau$-periodic motion that can be considered as the unperturbed, ideal tool motion when no self-excited vibrations arise, and $\xi(t)$ is the perturbation. Substitution of Eq. (10) into Eq. (9) yields:

$$
\begin{gathered}
\ddot{x}_{p}(t)+2 \zeta \omega_{n} \dot{x}_{p}(t)+\omega_{n}^{2} x_{p}(t)+\ddot{\xi}(t)+2 \zeta \omega_{n} \dot{\xi}(t) \\
+\omega_{n}^{2} \xi(t)=-\frac{b K_{s}(t)}{m}[\xi(t)-\xi(t-\tau)]-\frac{b f_{0}(t)}{m} .
\end{gathered}
$$

In the ideal case $\xi \equiv 0$, this gives

$\ddot{x}_{p}(t)+2 \zeta \omega_{n} \dot{x}_{p}(t)+\omega_{n}^{2} x_{p}(t)=-\frac{b f_{0}(t)}{m}$.

Since the excitation term $b f_{o}(t) / m$ is $\tau$-periodic, the particular solution of Eq. (12) is also $\tau$-periodic. This proves the existence of a $\tau$-periodic $x_{p}(t)$ and verifies the assumed form of Eq. (10). Now, the equation of motion is reduced to

$$
\begin{aligned}
& \ddot{\xi}_{(t)}+2 \zeta \omega_{n} \dot{\xi}(t)+\omega_{n}^{2} \xi(t) \\
& =-\frac{b K_{s}(t)}{m}[\xi(t)-\xi(t-\tau)] .
\end{aligned}
$$

This is the variational system of Eq. (9) about the $\tau$ periodic ideal motion $x_{p}(t)$. In other words, the stability of the $\xi(t) \equiv 0$ solution of Eq. (12) gives the stability of the $\tau$-periodic ideal solution $x_{p}(t)$ of Eq. (9). The term $K_{s}(t)$ in Eq. (13) is often called specific cutting force variation.
Eq. (13) is the standard linear non-autonomous DDE model of the milling process. The stability properties are determined by the eigenvalues of the monodromy operator.

\section{Up-milling and down-milling}

The relationship between the direction of tool rotation and feed defines two types of partial immersion milling operations: the up-milling and the down-milling (see Fig. 3 ). Both operations essentially produce the same result, but the dynamics and stability properties are not the same. Partial immersion milling operations are characterized by the number $N$ of teeth and the radial immersion ratio $a / D$, where $a$ is the radial depth of cut, $D$ the diameter of the tool.

Fig. 4 presents the specific cutting force variation $K_{s}(t)$ for different partial and full immersion up-milling and down-milling operations by a single fluted tool. The following experimentally identified parameters were used: $m=2.573 \mathrm{~kg}, \quad K_{n}=2.0 \times 10^{8} \mathrm{~N} / \mathrm{m}^{2} \quad$ and $K_{t}=$ $5.5 \times 10^{8} \mathrm{~N} / \mathrm{m}^{2}$ (see Part 2 of this report).

In the case of partial immersion milling, the specific cutting force variation is different for up- and down-milling operations. Obviously, the slotting (full immersion milling) is the same for both up- and down-milling, since these cases are mirror images of each other.

\section{Finite element analysis in time}

The stability of the milling process is dependent upon the perturbation growth or decay about the periodic motion determined by Eq. (13). Since this equation does not have a closed form solution, an approximate solution is sought to understand the behaviour of the system. One such approximation technique used for dynamic systems is time finite elements [32]. This method was first applied to an interrupted turning process by Halley [33] and Bayly et al. [34]. The authors matched an approxi- (a)

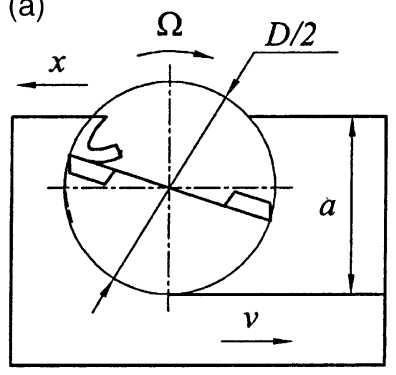

(b)

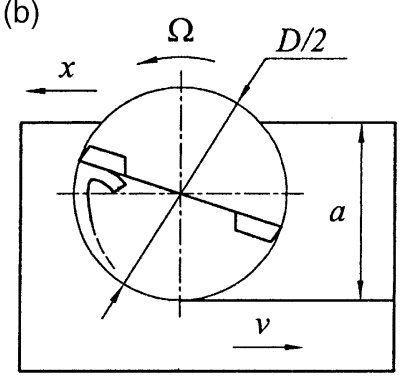

Fig. 3. Up-milling (a) and down-milling (b). 
up-milling

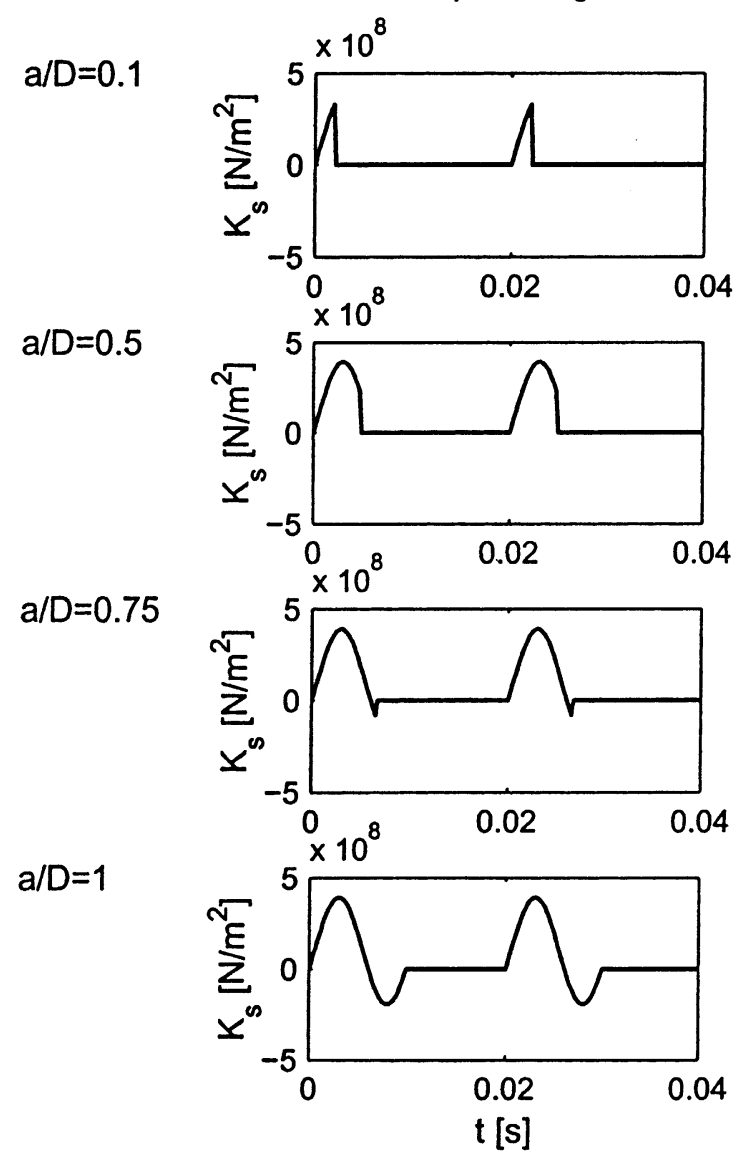

down-milling
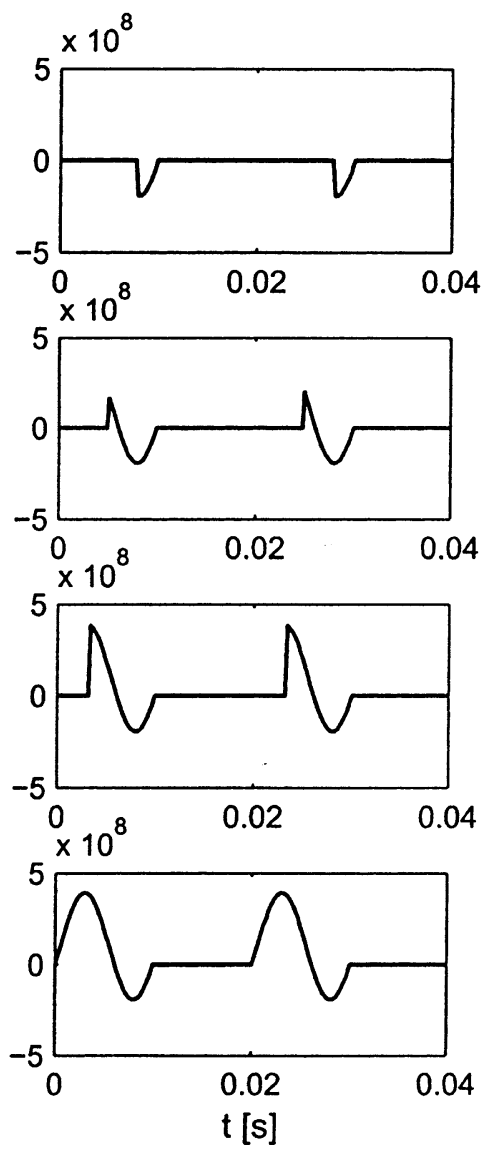

Fig. 4. The specific cutting force variation.

mation for the cutting motion obtained with a single finite element to the exact solution for free vibration to obtain a discrete map. The eigenvalues of the map were used to determine the stability of the system. Comparisons with experimental tests showed strong agreement for small fractions of the spindle period in the cut $\rho$. However, the authors noticed diminished correlation for larger values of $\rho$. This was corrected by Bayly et al. [22] by dividing the time in the cut into multiple finite elements in time. The analysis presented in this section adapts the method developed by Bayly et al. [22] for an interrupted turning process to model milling more closely by including the changing direction of cutting forces.

When the tool is not in contact with the workpiece, the system experiences free vibration:

$\ddot{\xi}(t)+2 \zeta \omega_{n} \dot{\xi}(t)+\omega_{n}^{2} \xi(t)=0$,

which has the solution $\xi(t)=c_{1} \mathrm{e}^{\lambda_{1} t}+c_{2} \mathrm{e}^{\lambda_{2} t}$, where $\lambda_{1,2}=-\zeta \omega_{n} \pm i \omega_{d}$ and $\omega_{d}=\omega_{n} \sqrt{1-\zeta^{2}}$ is the damped natural angular frequency. If we let $t=t_{c}$ as the tool leaves the material and $t_{f}$ be the duration of free vibration, a state transition matrix is obtained that relates the final state of free vibration to the initial state as in Bayly et al. [22]:

$$
\begin{aligned}
& \left(\begin{array}{l}
\xi\left(t_{c}+t_{f}\right) \\
\dot{\xi}\left(t_{c}+t_{f}\right)
\end{array}\right)=\frac{1}{\lambda_{1}-\lambda_{2}} \\
& \left(\begin{array}{ll}
\lambda_{1} \mathrm{e}^{\lambda_{2} t_{f}-\lambda_{2}} \mathrm{e}_{1}^{\lambda_{1} t_{f}} & \mathrm{e}^{\lambda_{1} t_{f}-\mathrm{e}^{\lambda_{2} t_{f}}} \\
\lambda_{1} \lambda_{2} \mathrm{e}^{\lambda_{2} t_{f}-} \lambda_{1} \lambda_{2} \mathrm{e}^{\lambda_{1} t_{f}} & \lambda_{1} \mathrm{e}^{\lambda_{1} t_{f}-} \lambda_{2} \mathrm{e}^{\lambda_{2} t_{f}}
\end{array}\right)\left(\begin{array}{l}
\xi\left(t_{c}\right) \\
\dot{\xi}\left(t_{c}\right)
\end{array}\right) .
\end{aligned}
$$

This equation is true for every period, such that for all $n$ :

$$
\left(\begin{array}{l}
\xi(n \tau) \\
\dot{\xi}(n \tau)
\end{array}\right)=\boldsymbol{\phi}\left(\begin{array}{l}
\xi\left((n-1) \tau+t_{c}\right) \\
\dot{\xi}\left((n-1) \tau+t_{c}\right)
\end{array}\right),
$$

where $\boldsymbol{\Phi}$ is the $2 \times 2$ matrix in Eq. (15).

When the tool is in the cut, the perturbation about the periodic tool motion is described by Eq. (13). Since this equation does not have a closed form solution, an approximate solution for the displacement of the tool during the $j^{\text {th }}$ element of the $n^{\text {th }}$ period of revolution is assumed in the following form [22]:

$\xi(t)=\sum_{i-1}^{4} a_{j i}^{n} \phi_{i}\left(\sigma_{j}(t)\right)$

Here $\sigma_{j}(t)=t-n \tau-\sum_{k=1}^{j-1} t_{k}$ is the "local" time within the $j^{\text {th }}$ element of the $n^{\text {th }}$ period, the length of the $k^{\text {th }}$ element is $t_{k}$ and the trial functions $\phi_{i}\left(\sigma_{j}\right)$ are the cubic 
Hermite polynomials. On the $j^{\text {th }}$ element these functions are:

$$
\begin{aligned}
& \phi_{1}\left(\sigma_{j}\right)=1-3\left(\frac{\sigma_{j}}{t_{j}}\right)^{2}+2\left(\frac{\sigma_{j}}{t_{j}}\right)^{3} \\
& \phi_{2}\left(\sigma_{j}\right)=t_{j}\left[\left(\frac{\sigma_{j}}{t_{j}}\right)-2\left(\frac{\sigma_{j}}{t_{j}}\right)^{2}+\left(\frac{\sigma_{j}}{t_{j}}\right)^{3}\right] \\
& \phi_{3}\left(\sigma_{j}\right)=3\left(\frac{\sigma_{j}}{t_{j}}\right)^{2}-2\left(\frac{\sigma_{j}}{t_{j}}\right)^{3} \\
& \phi_{4}\left(\sigma_{j}\right)=t_{j}\left[-\left(\frac{\sigma_{j}}{t_{j}}\right)^{2}+\left(\frac{\sigma_{j}}{t_{j}}\right)^{3}\right] .
\end{aligned}
$$

These functions are particularly useful because they allow the coefficients of the assumed solution to be found by matching the initial and final velocities for each element.

The initial conditions are

$$
\xi\left(t_{0 j}^{n}\right)=a_{01}^{n}, \quad \dot{\xi}\left(t_{o j}^{n}\right)=a_{j 2}^{n},
$$

and the final conditions are

$$
\xi\left(t_{1 j}^{n}\right)=a_{j 3}^{n}, \quad \dot{\xi}\left(t_{1 j}^{n}\right)=a_{j 4}^{n},
$$

where

$t_{0 j}^{n}=\left(n \tau+\sum_{k=1}^{j-1} t_{k}\right), t_{1 j}^{n}=\left(n \tau+\sum_{k=1}^{j} t_{k}\right)$

Substitution of the assumed solution into the equation of motion (13) leads to a non-zero error. This equation may be applied to the $j^{\text {th }}$ element:

$$
\begin{aligned}
& \left(\sum_{i=1}^{4} a_{j 1}^{n} \ddot{\phi}_{i}(t)\right)+2 \zeta \omega_{n}\left(\sum_{i=1}^{4} a_{j i}^{n} \dot{\phi}_{i}(t)\right) \\
& \quad+\omega_{n}^{2}\left(\sum_{i=1}^{4} a_{j i}^{n} \phi_{i}(t)\right)+b \frac{K_{s}(t)}{m}\left[\left(\sum_{i=1}^{4} a_{j i}^{n} \phi_{i}(t)\right)\right. \\
& \left.-\left(\sum_{i=1}^{4} a_{j i}^{n-1} \phi_{i}(t)\right)\right]=\varepsilon(t) .
\end{aligned}
$$

In the method of weighted residual, the error $\epsilon(t)$ is "weighted" by a set of test functions $\psi_{p}\left(\sigma_{j}\right), p=1,2$ and the integral of the weighted error is set to zero to obtain two more equations per element [35,22]. The test functions are chosen to be the simplest possible functions: $\psi_{1}\left(\sigma_{j}\right)=1$ and $\psi_{2}\left(\sigma_{j}\right)=\sigma_{j} / t_{j}-1 / 2$. The integral is taken over the time interval for each element, $t_{j}=$ $t_{c} / E$, thereby dividing the time in the cut $t_{c}$ into $E$ elements. The resulted two equations are thus

$$
\begin{aligned}
& \int_{0}^{t_{j}}\left[\left(\sum_{i=1}^{4} a_{j i}^{n} \ddot{\phi}_{i}\left(\sigma_{j}\right) \psi_{p}\left(\sigma_{j}\right)\right)\right. \\
& +2 \zeta \omega_{n}\left(\sum_{i=1}^{4} a_{j i}^{n} \dot{\phi}_{i}\left(\sigma_{j}\right) \psi_{p}\left(\sigma_{j}\right)\right) \\
& \left.+\omega_{n}^{2}\left(\sum_{i=1}^{4} a_{j i}^{n} \dot{\phi}_{i}\left(\sigma_{j}\right) \psi_{p}\left(\sigma_{j}\right)\right)\right] \mathrm{d} \sigma_{j} \\
& +b \int_{0}^{t_{j}} \frac{K_{s}\left(\sigma_{j}\right)}{m}\left[\left(\sum_{i=1}^{4} a_{j i}^{n-1} \dot{\phi}_{i}\left(\sigma_{j}\right) \psi_{p}\left(\sigma_{j}\right)\right)\right. \\
& \left.-\left(\sum_{i=1}^{4} a_{j i}^{n-1} \dot{\phi}_{i}\left(\sigma_{j}\right) \psi_{p}\left(\sigma_{j}\right)\right)\right] \mathrm{d} \sigma_{j}=0, \quad p=1,2,
\end{aligned}
$$

where $K_{s}\left(\sigma_{j}\right)$ has been used in place of the previously defined $K_{s}(t)$ to show dependence on the local time.

The displacement and velocity of the tool at the entry into the cut are specified by the coefficients of the first two basis functions on the first element: $a_{11}^{n}$ and $a_{12}^{n}$. The relationship between the initial and final conditions during free vibration can be rewritten in terms of the coefficients as:

$\left(\begin{array}{l}a_{11} \\ a_{12}\end{array}\right)^{n}=\boldsymbol{\Phi}\left(\begin{array}{l}a_{E 3} \\ a_{E 4}\end{array}\right)^{n-1}$,

where $E$ is the total number of finite elements in the cut. For the remainder of the elements, the position and velocity at the end of one element are equal to the position and velocity at the beginning of the next element:

$\left(\begin{array}{c}a_{j 1} \\ a_{j 2}\end{array}\right)^{n}=\left(\begin{array}{l}a_{(j-1) 3} \\ a_{(j-1) 4}\end{array}\right)^{n}$.

Eqs. (23) and (25) can be arranged into a global matrix relating the coefficients of the assumed solution in terms of the coefficients of the previous tooth passage. The following expression is for the case when the number of elements is $E=2$ :

$$
\left(\begin{array}{llllll}
1 & 0 & 0 & 0 & 0 & 0 \\
0 & 1 & 0 & 0 & 0 & 0 \\
N_{11}^{1} & N_{12}^{1} & N_{13}^{1} & N_{14}^{1} & 0 & 0 \\
N_{21}^{1} & N_{22}^{1} & N_{23}^{1} & N_{24}^{1} & 0 & 0 \\
0 & 0 & N_{11}^{2} & N_{12}^{2} & N_{13}^{2} & N_{14}^{2} \\
0 & 0 & N_{21}^{2} & N_{22}^{2} & N_{23}^{2} & N_{24}^{2}
\end{array}\right)\left(\begin{array}{l}
\left(\begin{array}{l}
a_{11} \\
a_{12}
\end{array}\right) \\
\left(\begin{array}{l}
a_{21} \\
a_{22}
\end{array}\right) \\
\left(\begin{array}{l}
a_{23} \\
a_{24}
\end{array}\right)
\end{array}\right)^{n}
$$




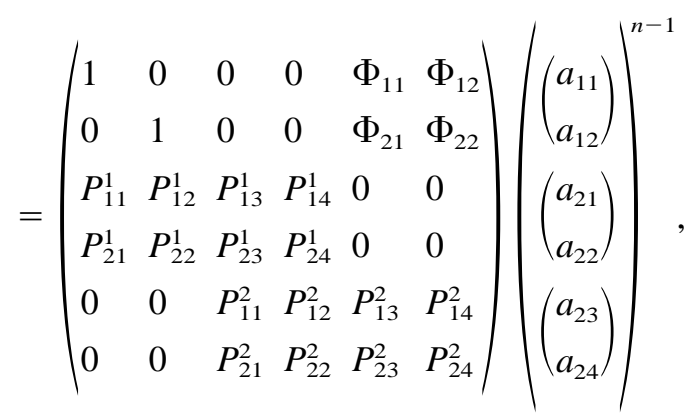

where $\Phi_{11}, \Phi_{12}, \Phi_{21}, \Phi_{22}$ are the elements of $\boldsymbol{\Phi}$ in Eq. (16), and

$$
\begin{aligned}
& N_{p i}^{j}=\int_{0}^{t_{j}}\left[\ddot{\phi}_{i}\left(\sigma_{j}\right)+2 \zeta \omega_{n} \dot{\phi}_{i}\left(\sigma_{j}\right)\right. \\
& \left.+\left(\omega_{n}^{2}+\frac{b K_{s}\left(\sigma_{j}\right)}{m}\right) \phi_{i}\left(\sigma_{j}\right)\right] \psi_{p}\left(\sigma_{j}\right) \mathrm{d} \sigma_{j},
\end{aligned}
$$

$P_{p i}^{j}=\int_{0}^{t_{j}} \frac{b K_{s}\left(\sigma_{j}\right)}{m} \phi_{i}\left(\sigma_{j}\right) \psi_{p}\left(\sigma_{j}\right) \mathrm{d} \sigma_{j}$.

It should be noted that the indices for $N_{p i}^{j}$ and $P^{j}{ }_{p i}$ give the location of these terms in Eq. (27) for higher $E$ as well.

Eq. (26) describes a discrete dynamic system, or map, that can be written as

$\mathbf{A} \mathbf{a}_{n}=\mathbf{B} \mathbf{a}_{n-1}$,

or

$\mathbf{a}_{n}=\mathbf{Q} \mathbf{a}_{n-1}$.

The eigenvalues of the transition matrix $\mathbf{Q}=\mathbf{A}^{-1} \mathbf{B}$ determine the stability of the system. If the eigenvalues for a given depth of cut $b$ and spindle speed $\Omega$ are in a modulus less than one, the milling process is asymptotically stable. If the magnitude of any eigenvalue exceeds one then the process is unstable.

The chatter frequencies for an unstable cutting process are also determined by the eigenvalues $\mu$ of the transition matrix Q, as it was shown by Insperger et al. [36]. These eigenvalues are also called characteristic multipliers. For the case of Hopf bifurcations, the critical eigenvalue has the form $\mu=\mathrm{e}^{ \pm \mathrm{i} \omega \tau}$. The chatter frequencies in this region are given by

$$
\begin{aligned}
& f_{H}=\left\{ \pm \omega+n \frac{2 \pi}{\tau}\right\}[\mathrm{rad} / \mathrm{s}] \\
& =\left\{ \pm \frac{\omega}{2 \pi}+n \frac{N \Omega}{60}\right\}[\mathrm{Hz}], \quad n=\ldots,-1,0,1, \ldots,
\end{aligned}
$$

where the angular chatter frequency is $\omega=\operatorname{Im}(\operatorname{In} \mu) / \tau$ with the restriction $\omega \in(0,2 \tau)$.

In the case of a period doubling bifurcation, $\mu=-$ $1=\exp (\mathrm{i} \pi)$, consequently $\omega=\pi$, and the chatter frequencies are

$$
\begin{aligned}
& f_{P D}=\left\{\frac{\pi}{\tau}+n \frac{2 \pi}{\tau}\right\}[\mathrm{rad} / \mathrm{s}] \\
& =\left\{\frac{N \Omega}{30}+n \frac{N \Omega}{60}\right\}[\mathrm{Hz}], \quad n=\ldots,-1,0,1, \ldots,
\end{aligned}
$$

The frequencies coming from the tooth pass excitation effect are determined by

$f_{T P E}=\left\{n \frac{N \Omega}{60}\right\}[\mathrm{Hz}], \quad n=1,2, \ldots$.

The indices $H, P D$ and TPE refer to secondary Hopf, period doubling and tooth pass excitation, respectively.

\section{Semi-discretization method}

Discretization techniques are important for differential equations for which the solution cannot be given in closed forms. The so-called semi-discretization is a well known technique in the finite element analysis of solid bodies, or in computational fluid mechanics. The basic idea is, that the corresponding partial differential equation (PDE) is discretized along the spatial coordinates only, while the time coordinates are unchanged. From a dynamical systems viewpoint, the PDE has an infinite dimensional state space, which is approximated by the finite dimensional state space of a higher dimensional ODE.

Semi-discretization of a DDE means that the time delayed term is approximated by a piecewise constant function, while the current time domain terms are left in the original form. Thus, the DDE is approximated by a series of ODEs.

Rewrite Eq. (13) in the form

$$
\begin{aligned}
\ddot{\xi}(t) & +2 \zeta \omega_{n} \dot{\xi}(t)+\left[\omega_{n}^{2}+\frac{b K_{s}(t)}{m}\right] \xi(t) \\
= & \frac{b K_{s}(t)}{m} \xi(t-\tau) .
\end{aligned}
$$

Let us introduce the interval division $\left(t_{i}, t_{i+1}\right)$, $t_{i+1}-t_{i}=\Delta t, i \in Z$, of the time domain, so that $\tau=(M+$ $1 / 2) \Delta t$, where $M$ is a positive integer, and approximate the $\tau$-period DDE (34) by

$$
\begin{aligned}
& \ddot{\xi}(t)+2 \zeta \omega_{n} \dot{\xi}(t)+\left[\omega_{n}^{2}+\frac{b K_{s i}}{m}\right] \xi(t) \\
& =\frac{b K_{s i}}{m} \xi_{i-M}, \quad t \in\left[t_{i}, t_{i+1}\right), \quad i=0,1, \ldots,
\end{aligned}
$$

where $\xi_{i}=\xi\left(t_{i}\right)$ for all $i \in Z$, and 
$K_{s i}=\frac{1}{\Delta t} \int_{t_{i}}^{t_{i+1}} K_{s}(t) d t$.

Eq. (35) is also a DDE with a $\tau$-periodic, saw-like time delay $\tilde{\tau}$, as it is shown in Fig. 5. As the parameter $M \rightarrow 0$ while $\Delta t \rightarrow 0$, Eq. (35) converges to Eq. (34) (for details, see [31]).

Although Eq. (35) is a DDE, it also defines an autonomous linear ODE with constant excitation on the righthand side in each interval $\left(t_{i}, t_{i+1}\right), i \in Z$. For the initial conditions, $\xi\left(t_{i}\right)=\xi_{i}, \dot{\xi}\left(t_{i}\right)=\dot{\xi}_{i}$ the solution and its derivative at each time instant $t_{i+1}$ can be determined:

$\xi_{i+1}=\xi\left(t_{i+1}\right)=a_{00} \xi_{i}+a_{01} \dot{\xi}_{i}+b_{0 M} \xi_{i-M}$,

$\dot{\xi}_{i+1}=\dot{\xi}\left(t_{i+1}\right)=a_{10} \xi_{i}+a_{11} \dot{\xi}_{i}+b_{1 M} \xi_{i-M}$,

where

$a_{00}=\kappa_{10} \exp \left(\lambda_{1} \Delta t\right)+\kappa_{20} \exp \left(\lambda_{2} \Delta t\right)$,

$a_{01}=\kappa_{11} \exp \left(\lambda_{1} \Delta t\right)+\kappa_{21} \exp \left(\lambda_{2} \Delta t\right)$

$a_{10}=\kappa_{10} \lambda_{1} \exp \left(\lambda_{1} \Delta t\right)+k_{20} \lambda_{2} \exp \left(\lambda_{2} \Delta t\right)$

$a_{11}=\kappa_{11} \lambda_{1} \exp \left(\lambda_{1} \Delta t\right)+\kappa_{21} \lambda_{2} \exp \left(\lambda_{2} \Delta t\right)$

$b_{0 M}=\sigma_{1} \exp \left(\lambda_{1} \Delta t\right)+\sigma_{2} \exp \left(\lambda_{2} \Delta t\right)+\frac{K_{s i} b}{m \omega_{n}^{2}+K_{s i} b}$,

$b_{1 M}=\sigma_{1} \lambda_{1} \exp \left(\lambda_{1} \Delta t\right)+\sigma_{2} \lambda_{2} \exp \left(\lambda_{2} \Delta t\right)$,

and

$\lambda_{1,2}=\frac{-2 \zeta \omega_{n} \pm \sqrt{\left(2 \zeta \omega_{n}\right)^{2}-4\left(\omega_{n}^{2}+K_{s i} b / m\right)}}{2}$,

$k_{10}=\frac{\lambda_{2}}{\lambda_{2}-\lambda_{1}}, k_{11}=\frac{-1}{\lambda_{2}-\lambda_{1}}, \quad \sigma_{1}=\frac{-\lambda_{2}}{\lambda_{2}-\lambda_{1} m \omega_{n}^{2}+K_{s i} b}$,

$k_{20}=\frac{-\lambda_{1}}{\lambda_{2}-\lambda_{1}}, k_{21}=\frac{1}{\lambda_{2}-\lambda_{1}}, \quad \sigma_{2}=\frac{\lambda_{1} \quad K_{s i} b}{\lambda_{2}-\lambda_{1} m \omega_{n}^{2}+K_{s i} b}$.

Eqs. (37) and (38) defines the discrete map

$\mathbf{y}_{i+1}=\mathbf{B}_{i} \mathbf{y}_{i}$,

where the $M+2$ dimensional state vector is

$\mathbf{y}_{i}=\operatorname{col}\left(\dot{\xi}_{i} \xi_{i} \xi_{i-1} \ldots \xi_{i-\mathrm{M}}\right)$,

and the coefficient matrix has the form
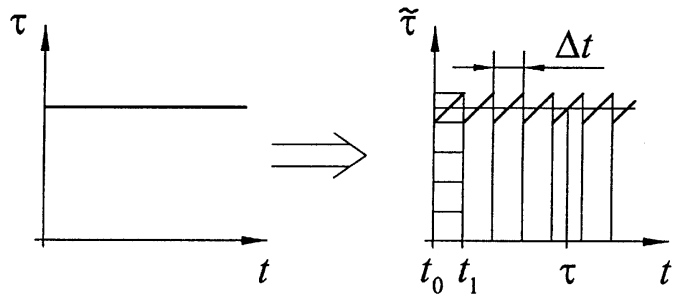

Fig. 5. Time delay approximation.

$$
\boldsymbol{B}_{i}=\left(\begin{array}{llllll}
a_{11} & a_{10} & 0 & \ldots & 0 & b_{1 M} \\
a_{01} & a_{00} & 0 & \ldots & 0 & b_{0 M} \\
0 & 1 & 0 & \ldots & 0 & 0 \\
\vdots & \vdots & \vdots & \ddots & \vdots & \vdots \\
0 & 0 & 0 & \ldots & 0 & 0 \\
0 & 0 & 0 & \ldots & 1 & 0
\end{array}\right) .
$$

Eq. (39) makes the connection between states at time $t_{i}$ and $t_{i+1}$.

The connection between the states at $t_{0}$ and $t_{0}+$ $k \Delta t=t_{k}$ is given by coupling of the coefficient matrices in each interval:

$\mathbf{y}_{k}=\mathbf{B}_{k-1} \mathbf{B}_{k-2} \ldots \mathbf{B}_{0} \boldsymbol{y}_{0}$.

The Floquet transition matrix can approximately be given by coupling the solutions of the first $M+1$ intervals:

$\boldsymbol{\Phi}=\mathbf{B}_{M} \mathbf{B}_{M-1} \ldots \mathbf{B}_{0}$,

since if $\Delta t$ is small enough and $M$ is large, then $(M+1) \Delta t \rightarrow \tau$. The stability properties are determined by the eigenvalues of $\boldsymbol{\Phi}$, that is, by the characteristic multipliers. If all these eigenvalues are in modulus less than one, than the process is asymptotically stable. The frequencies of the arising vibrations can be calculated by the same formulas as in Eqs. (31)-(33).

\section{Stability charts, comparison of methods}

Stability charts and chatter frequencies are determined by both methods for a series of milling processes. For the calculations, the experimentally identified parameters were used: $m=2.573 \mathrm{~kg}, \zeta=0.0032, f_{n}=\omega_{n} / 2 \pi=$ $146.5 \mathrm{~Hz}, \quad K_{n}=2.0 \times 10^{8} \mathrm{~N} / \mathrm{m}^{2}$ and $K_{t}=$ $5.5 \times 10^{8} \mathrm{~N} / \mathrm{m}^{2}$ (see Part 2 of this report).

In Fig. 6, stability charts and the chatter frequencies $f$ are presented for full immersion milling case. These frequencies are either $f_{H}$ or $f_{P D}$ determined by Eqs. (31) and (32), while the tooth pass excitation frequencies $F_{T P E}$ are not presented in these charts. In Fig. 6 (a), the lobes presented by continuous lines were obtained by the FEAT method with $E=2$ that results a $6 \times 6$ sized transition matrix. The lobes presented by dashed lines were obtained by the SD method with $M=8$ that results a $10 \times 10$ sized transition matrix. The comparison of the lobes obtained by the two methods show large deviations at some parameter ranges. In Fig. 6(b), the continuous curves were obtained by FEAT method with $E=4$ ( $10 \times 10$ sized transition matrix), the dashed curves were obtained by the SD method with $M=48(50 \times 50$ sized transition matrix). In this case, the differences in the stability predictions are slight and acceptable. 
(a)
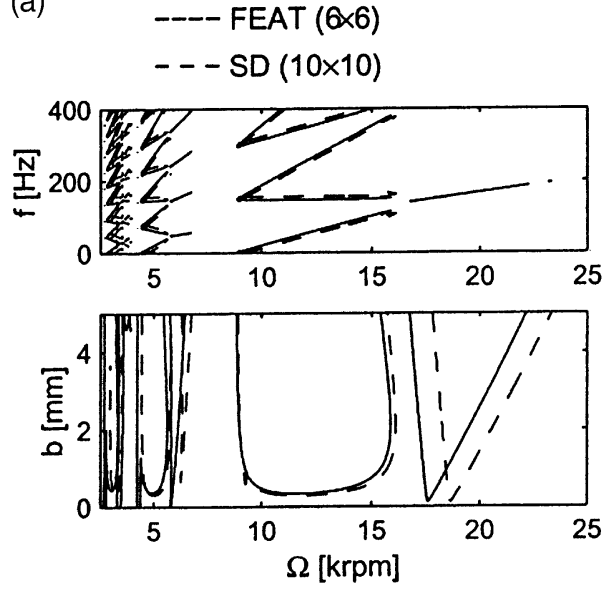

(b)

$$
\begin{aligned}
& --- \text { FEAT }(10 \times 10) \\
& ---S D(50 \times 50)
\end{aligned}
$$
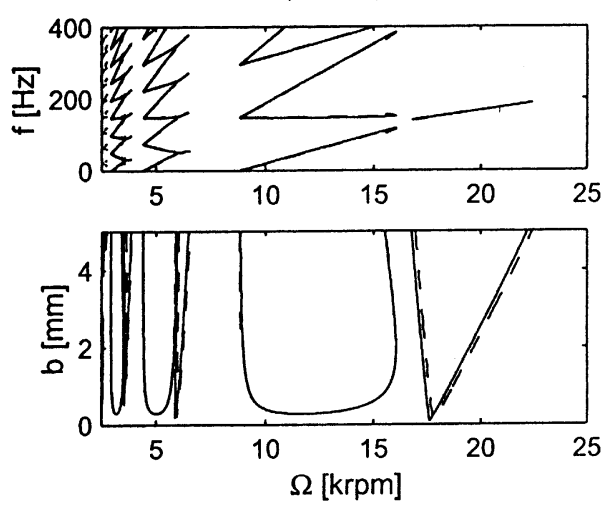

Fig. 6. Stability lobes and chatter frequencies for full immersion milling.

The size of the applied transition matrix gives a kind of base to compare the two methods. In the case of the FEAT method, the infinite dimensional DDE (13) is approximated by $6 \times 6$ and $10 \times 10$ dimensional discrete systems, in the case of the SD method, $10 \times 10$ and $50 \times 50$ dimensional discrete approximation is used.

The lobes shown in Fig. 6 show that the FEAT method is more efficient than the SD method. There are two basic reasons for this. First, the FEAT method contains a symbolic calculation that makes the procedure faster. Second, the FEAT method discretizes only the time in the cut, while the SD method discretizes all the time domain independently if it is in the cut or not. However, this effect is negligible for high immersion milling, when the time in the cut is large, and the SD method can be used for more general cases of distributed time delays or varying cutting speeds.

In Fig. 7, stability charts and the corresponding chatter frequencies are shown for $0.1,0.5$ and 0.75 immersion up- and down-milling. The continuous curves were obtained by the FEAT method with $10 \times 10$ sized transition matrix, the dashed curves were obtained by the SD method with $50 \times 50$ sized transition matrix. The curves show good agreement.

There are two types of stability lobes [36]: the ones related to secondary Hopf bifurcation, and the ones related to period doubling (or flip) bifurcations. These lobes are referred to as Hopf lobes and flip lobes, respectively. For the case of flip lobes, the chatter frequencies depend linearly on the spindle speed as it is shown by Eq. (32). For the Hopf lobes, the number of chatter frequencies are duplicated, since the relevant characteristic multipliers are a complex conjugate pair crossing the unit circle. This phenomenon is shown by both the FEAT and the SD methods. Independently from the applied approximation method, the physical consequences of the results are discussed in the subsequent concluding section.

\section{Conclusions}

In Fig. 7, the similarities and the differences between up-milling and down-milling cases can be clearly observed. The flip lobes, for example, vary in size, but they are located more or less at the same spindle speed ranges. This is not always true for the Hopf lobes. For low immersion up-milling, the Hopf lobes are located to the left of the flip lobes, while for down-milling, the Hopf lobes are positioned to the right of the flip lobes. Also, the frequency plots of up- and down-milling cases show this special duality or mirror symmetry for immersions $1 / 2$ and below.

The physical explanation for these surprising results is as follows. The flip lobes are related to the impact effects of entering and leaving the workpiece material. These are more or less independent of the sense (up or down) of the milling. This is not the case for the Hopf lobes. It is well known but rarely referred to, that the conventional stability chart of turning contains a part for negative depth of cut which has no physical meaning there (see Fig. 8). The corresponding dimensionless autonomous DDE model has the from

$\ddot{\xi}(t)+2 \zeta \dot{\xi}(t)+\xi(t)=-\frac{b K}{m \omega_{n}^{2}}[\xi(t)-\xi(t-\tau)]$,

where $b$ is the depth of cut, $K$ is the cutting coefficient of turning, $m, \zeta, \omega_{n}$ are model parameters and $\tau$ is the tooth pass period.

At the negative depth of cut region, the Hopf lobes are just in the dual regions of cutting speeds. These lobes may become relevant and be transformed into the positive depth of cut region for operations where specific cutting force variation $K_{s}(t)$ (or the corresponding cutting coefficient $K$ of turning) has a negative time-average value. This is just what happens in cases of half and less immersion down-milling as shown also in the upper right diagrams of Fig. 4. 
$a / D=0.1$
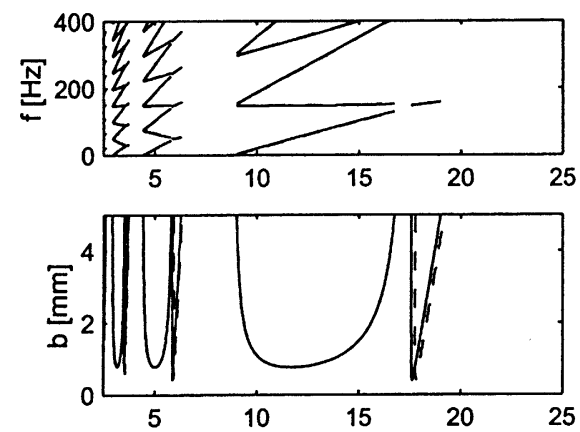

$a / D=0.5$
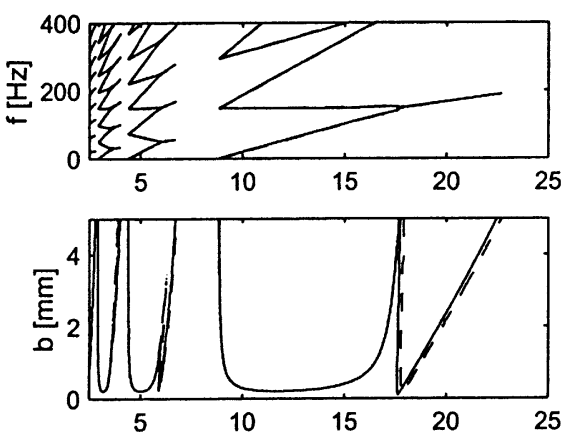

$\mathrm{a} / \mathrm{D}=0.75$
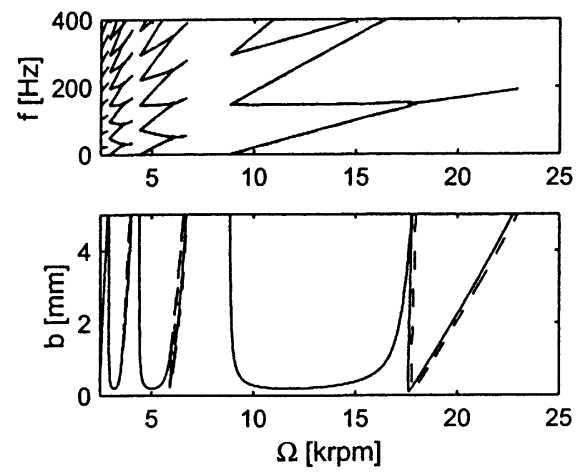

down-milling
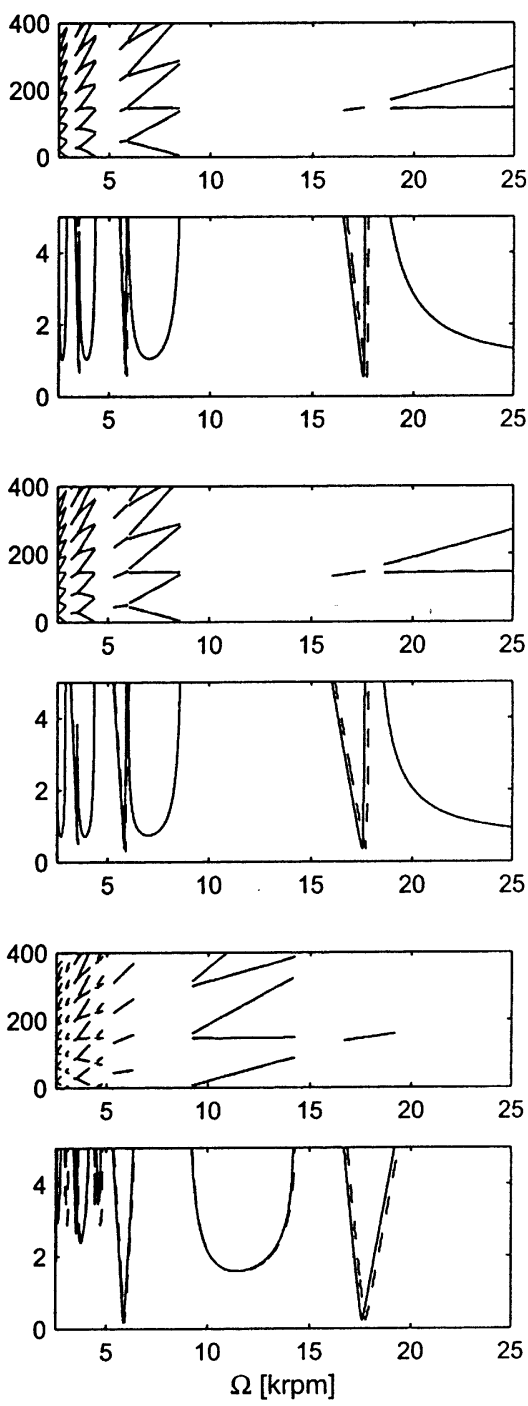

Fig. 7. Stability lobes and chatter frequencies for partial immersion up- and down-milling operations.

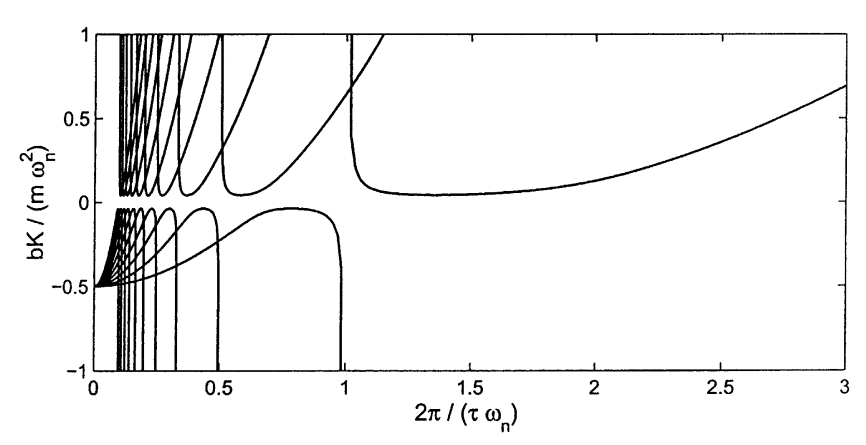

Fig. 8. Stability boundaries for Eq. (44).
These results are also confirmed experimentally as presented in Part 2 of this report. The practical relevance of these observations are obvious: high-speed milling operations can be stabilized simply by changing to down-milling from up-milling at certain wide high-speed parameter domains.

\section{Acknowledgements}

This research was supported by the Hungarian National Science Foundation under grant no. OTKA T030762, the US National Science Foundation Grants DMI-9900108 (GOALI) and CMS-9625161 (CAREER) 
and the US National Defense Science and Engineering Graduate Fellowship.

\section{References}

[1] J. Tlusty, Manufacturing Processes and Equipment, Prentice Hall, New Yersey, 2000.

[2] F.W. Taylor, On the art of cutting metals, Transactions of ASME 28 (1907) 31-350.

[3] J. Tlusty, A. Polacek, C. Danek, J. Spacek, Selbsterregte Schwingungen an Werkzeug-maschinen, VEB Verlag Technik, Berlin, 1962.

[4] S.A. Tobias, Machine Tool Vibration, Blackie, London, 1965.

[5] V.A. Kudinov, Theory of vibration generated from metal cutting (in Russian), New Technology of Mechanical Engineering, USSR Academy of Sciences Publishing House, Moscow, 1955.

[6] V.A. Kudinov, Dynamics of Tool-Lathe (in Russian), Mashinostroenie, Moscow, 1967.

[7] F.C. Moon, Dynamics and Chaos in Manufacturing Processes, Wiley, New York, 1998.

[8] D.J. Seagalman, E.A. Butcher, Suppression of regenerative chatter via impendance modulation, Journal of Vibration and Control 6 (2000) 243-256.

[9] G. Stépán, Modelling nonlinear regenerative effects in mental cutting, Philosophical Transactions of the Royal Society 359 (2001) 739-757.

[10] A.M. Gouskov, S.A. Voronov, H. Paris, S.A. Batzer, Cylindrical workpiece turning using multiple-cutter tool heads, Proceedings of the ASME 2001 Design Engineering Technical Conferences, Pittsburgh, Pennsylvania (2001) paper no. DETC2001/VIB21431 (CD-ROM).

[11] I. Minis, R. Yanushevsky, A new theoretical approach for the prediction of machine tool chatter in milling, Journal of Engineering for Industry 115 (1993) 1-8.

[12] G. Stépán, Retarded Dynamical Systems, Longman, Harlow, 1989.

[13] H.M. Shi, S.A. Tobias, Theory of finite amplitude machine tool instability, International Journal of Machine Tool Design and Research 24 (1984) 45-69.

[14] G. Stépán, T. Kalmár-Nagy, Nonlinear regenerative machine tool vibration, Proceedings of the 1997 ASME Design Engineering Technical Conferences, Sacramento, California (1997) paper no. DETC97/VIB-4021 (CD-ROM).

[15] A. Halanay, Stability theory of linear periodic systems with delay (in Russian), Revue de Mathéematiques Pures et Appliquées 6 (4) (1961) 633-653.

[16] J.K. Hale, Theory of Functional Differential Equations, SpringerVerlag, New York, 1977.

[17] Y. Altintas, E. Budak, Analytical prediction of stability lobes in milling, Annals of the CIRP 44 (1) (1995) 357-362.

[18] E. Budak, Y. Altintas, Analytical prediction of chatter stability in milling - Part I: General formulation, Journal of Dynamic Systems, Measurement and Control 120 (1998) 22-30.

[19] E. Budak, Y. Altintas, Analytical prediction of chatter stability in milling-Part II: Application of the general formulation to common milling systems, Journal of Dynamic Systems, Measurement and Control 120 (1998) 31-36.

[20] M.A. Davies, J.R. Pratt, B. Dutterer, T.J. Burns, Stability prediction for low radial immersion milling, Journal of Manufacturing Science and Engineering 124 (2) (2002) 217-225.

[21] T. Insperger, G. Stépán, Stability of high-speed milling, Proceedings of the ASME 2000 DETC, Symposium on Nonlinear Dynamics and Stochastic Mechanics, Orlando, Florida, AMD241 (2000) 119-123.

[22] P.V. Bayly, J.E. Halley, P.B. Mann, M.A. Davies, Stability of interrupted cutting by temporal finite element analysis, Proceedings of the ASME 2001 Design Engineering Technical Conferences, Pittsburgh, Pennsylvania (2001) paper no. DETC2001/VIB-21581. (CD-ROM).

[23] J. Tian, S.G. Hutton, Chatter instability in milling systems with flexible rotating spindles-a new theoretical approach, Journal of Manufacturing Science and Engineering 123 (1) (2001) 1-9.

[24] S. Smith, J. Tlusty, An overview of modeling and simulation of the milling process, Journal of Engineering for Industry 113 (1991) 169-175.

[25] B. Balachandran, Non-linear dynamics of milling process, Philosophical Transactions of the Royal Society 359 (2001) 793-820.

[26] M.X. Zhao, B. Balachandran, Dynamics and stability of milling process, International Journal of Solids and Structures 38 (10-13) (2001) 2233-2248.

[27] D.M. Esterling, Y. Ren, Y.S. Lee, Time-domain chatter prediction for high speed machining, Proceedings of 2002 Annual North American Manufacturing Research Conference (NAMRC XXX), West Lafayette, Indiana (2002) in press.

[28] T.L. Schmitz, M.A. Davies, K. Medicus, J. Snyder, Improving high-speed machining material removal rates by rapid dynamic analysis, Annals of the CIRP 50 (1) (2001) 263-268.

[29] W.T. Corpus, W.J. Endres, A high-order solution for the added stability lobes in intermittent machining, Proceedings of the ASME 2001 DETC, Symposium on Machining Processes, Orlando, Florida, MED-11 (2000) 871-878.

[30] G. Stépán, R. Szalai, Nonlinear vibrations of highly interrupted machining, Proceedings of the 2nd Workshop of COST P4 WG2 on Dynamics and Control of Mechanical Processing, Budapest, Hungary (2001) 59-64.

[31] T. Insperger, G. Stépán, Semi-discretization method for delayed systems, International Journal for Numerical Methods in Engineering (2002) in press.

[32] L. Meirovitch, Elements of Vibration Analysis, McGraw-Hill, Inc., New York, 1986

[33] J. E. Halley, Stability of low radial immersion milling, MSc Thesis, Washington University, St. Louis, Missouri, 1999.

[34] P.V. Bayly, J.E. Halley, M.A. Davies, J.R. Pratt, Stability analysis of interrupted cutting with finite time in the cut, Proceeding of the ASME 2000 DETC, Manufacturing in Engineering Division, Orlando, Florida, MED-11 (2000) 989-994.

[35] D.A. Peters, A.P. Idzapanah, Hp-version finite elements for the space-time domain, Computational Mechanics 3 (1988) 73-88.

[36] T. Insperger, G. Stépán, P.V. Bayly, B.P. Mann, Multiple chatter frequencies in milling processes, Journal of Sound and Vibration (2002) in press. 